\title{
Outcomes Research in Facial Plastic Surgery: A Review and New Directions. An Update
}

\author{
Ramsey Alsarraf ${ }^{1,2}$
}

Published online: 5 August 2020

(C) Springer Science+Business Media, LLC, part of Springer Nature and International Society of Aesthetic Plastic Surgery 2020

As we celebrate the 50th anniversary of the ISAPS, and revisit selected contributions of its affiliated journal Aesthetic Plastic Surgery, it is interesting to see the range of most cited articles during this history, and with pleasure that I update why "Outcomes Research in Facial Plastic Surgery: A Review and New Directions" is as relevant today as it was twenty years ago when it was first published. One can see that the majority of these articles that are being revisited focus on specific surgical procedures, many of which remain to this day truly landmark improvements in plastic surgery technique. Just as other reviews of the last 50-70 years have shown, whether it is the evolution of deep plane, SMAS, or endoscopic face-lift techniques, a better understanding of facial anatomy and its implications for safer approaches to the eyelids, face, or neck, or improvements in perioperative and adjuvant therapies, such articles represent truly innovative technical improvements that make plastic surgery procedures more consistent, less risky, and more attractive to our patient population as a whole. The articles, like mine, which focus instead on the field of plastic surgery from without, the way in which results are compared and outcomes determined, in some ways simply compliment those technical advances which have driven our specialty over the last half century. How is one to know if technique $A$ is truly better than technique $\mathrm{B}$, if one cannot measure the outcomes of these two procedures in some quantitative and reproducible way?

Ramsey Alsarraf

info@thenewburycenter.com

1 The Newbury Center for Cosmetic Facial Plastic Surgery, 69 Newbury Street, Boston, USA

2 Massachusetts Eye \& Ear Infirmary, Harvard Medical School, Boston, MA, USA
Twenty years ago, when this original article was first published, the answer to this question was difficult to find. Each of us, as surgeons, knows when our patients are completely happy and when they have misgivings; we know when a surgery has been a total, home-run success, and when it, unfortunately, falls short. The purpose of this original article was to suggest a replacement for these anecdotal and qualitative feelings of success or failure with the measurable and quantitative tools of quality-of-life outcomes research.

And so, did we listen? In fact, a simple PubMed search reveals how dramatically times have indeed changed during the last twenty years. During the twenty years prior to the publication of this article, from 1980-2000, there were a total of 225 articles on PubMed that discuss both the terms "outcomes" and "plastic surgery," and a mere 183 specifically found using both the terms "quality of life" and "plastic surgery" as well. In contrast, in the twenty years subsequent to this publication, from 2000-2020, "quality of life" and "plastic surgery" articles total 2872, and "outcomes" and "plastic surgery" articles now top 12,000 . Obviously, I in no way mean to imply that this simple, somewhat tentative and very preliminary review article, is in any way the cause of this increase but it does, however, reflect the timeliness of its publication in Aesthetic Plastic Surgery as a journal ahead of its time, as well as the atmosphere of academic interest from which the well-spring of plastic surgery research during the last twenty years has certainly benefited. In some ways, such an increase is simply semantic. Because the new field of the so-called outcomes research was coming to the forefront in all fields of medicine and surgery at the turn of the century, many researchers since then have simply incorporated the terms of its use in their otherwise typical studies of 
procedure $\mathrm{X}$, medicine $\mathrm{Y}$, or technique $\mathrm{Z}$. And thus, words such as "outcomes" and "quality of life" abound. But, in addition to this semantic point, what is truly encouraging is the manner in which researchers have embraced the actual spirit of outcomes research, with its ever-present focus on the hitherto subjective, patient-based results of one procedure, medicine, or technique over another.

In the 1970s, 1980s, and 1990s, there were only a handful of questionnaires measuring quality-of-life outcomes in general, and just one or two specifically designed to measure the outcomes of the cosmetic plastic surgery procedures which we perform day in and day out as cosmetic plastic surgeons. Now, there are literally hundreds of general QOL instruments, and dozens of reliable, validated, and pilot-tested disease-specific instruments for these same procedures. In rhinoplasty alone, it is far more commonplace to read a study that employs one of the many available QOL instruments as its measure of outcomes, than to find one that does not. There is the ROE, the RHINO, the NOSE, and the SCHNOS, to name but a few, many of which have been translated and validated in various languages and countries around the world. Notwithstanding their sometimes unfortunate names, this plethora of available disease-specific outcomes questionnaires surely indicates just how fortunate a time it is for those in search of a more evidence-based, quantitative assessment of outcomes in cosmetic facial plastic surgery, and plastic surgery in general. And, in fact, this was precisely the hope of my own original article. It should not matter which instrument one chooses to use to measure the success of a rhinoplasty procedure, which questionnaire one employs to evaluate the results of a certain face-lift technique, or which QOL measure one incorporates to compare skin-resurfacing options, so long as we continue, as a specialty, to use something. Some method measures patient satisfaction as an end, in and of itself. And so, we can still compare how many millimeters of tip rotation a certain cartilage graft technique yields over another, or how many degrees of elevation a certain face-lift plane provides, or how many brown spots per inch a given laser removes, but we can also, now, determine whether or not those rotated millimeters or elevated degrees or removed spots translate to our patients actually being happier.

While we reflect on the last fifty years and revisit these often seminal articles once again, I would suggest that we also look forward, toward the next fifty years and where our specialty of cosmetic plastic surgery is headed. How will our techniques and procedures continue to evolve and improve, which of the so-called advances are truly improvements, and which are simply passing fads that some will embrace and some will avoid? Will noninvasive, threading, or endoscopic techniques hold their place in our skillful armamentarium of surgical techniques, do fat transfers and repositioning remain a go-to aspect of our procedures, and is every new filler, dermatologic tightening machine, or injectable a must-have option for our aesthetic patients? On a personal note, after twenty years in practice here in Boston, will I myself really stray from the deep plane face and neck-lift, coronal brow-lift, trans-conjunctival blepharoplasty, or open rhinoplasty techniques in which I was trained and upon which I have built my own reputation? I suspect that I will not. But I do hope, regardless of how each of our own, personal surgical techniques change, evolve, or remain the same, that each of us continues to embrace the notion that the way in which we measure the outcomes of these techniques does matter and does, indeed, need to continue to improve. Any evidence-based field of medicine simply depends on it.

Publisher's Note Springer Nature remains neutral with regard to jurisdictional claims in published maps and institutional affiliations. 